\title{
Artificial Intelligence Based A* Optimization Routing in Mobile Ad Hoc Networks
}

\author{
Gondi Yasoda Devi, Gurrala Venkateswara Rao
}

\begin{abstract}
A Mobile Ad Hoc Network (MANET) is a hotchpotch of nodes with mobility feature, the established network utilization is dynamically outlined based on temporary architecture. In MANETs, the challenging and vital role is played by the routing protocols performance factors under different condition and environments. The routing protocols are liable to handle many nodes with limited resources. There exits many routing protocols in MANETs, one of the main key note that has to be considered in designing a routing protocol is to observe that the designed routing protocol is having an proportionate effect on network performance. The existence of obstacles may lead to many geographical routing problems like excess consumption of power and congestion of data. The aim of this paper is to take the assistance of $A^{*}$ algorithm that finds the walk-able path avoiding the concave obstacle in the path relaying on the gaming-theory model[29]. This algorithm decreases the delays in packet transmission and in turn increases the success rate of transmission. We take into consideration path length, penalty for node availability as probability of forwarding criteria and processes effective packet transmission. The simulated results analyse the performance of our protocol over other conventional algorithms based on congestion cost, path length, node availability penalty, delay, packet loss, throughput.
\end{abstract}

KEYWORDS: MANETS, A*, Penalty of Node Availability, Path Length, Heuristic.

\section{INTRODUCTION}

$W_{\text {ireless communication is topmost communication }}$ channel of this era form top to bottom in any part of the world. Several challenges has to be taken into consideration while enhancing the technology. One of the most dynamic technology we are very fond off, in communication, without which we feel like our hand are cut is the Mobile adhoc networks (MANETs), where a researcher has to pay a key role in designing the optimistic routing protocols. In wireless communication MANETs are self regulating automated networks. In MANETs the nodes are by nature having the feature of mobility they do not relay on infrastructure and nodes communicate and interact with each other across the wireless the interfaces [9]. MANETs are easily applicable when there is a requirement for temporary communication system without infrastructure for instance events of large wild and region stricken by earthquakes [10].

Revised Manuscript Received on December 30, 2019.

* Correspondence Author

Gondi Yasoda Devi*, Research Scholar, Department of CSE

GIT, GITAM Deemed to be University, Visakhapatnam, India (Faculty Member Department of CSE, Lendi Institute of Engineering \& Technology, Vizianagaram) E-mail:gondi.yasoda@gmail.com

Dr. Gurrala Venkateswara Rao, Department of CSE, GIT, GITAM Deemed to be University, Visakhapatnam, India.

E- mail:venkateswararao.gurrala@gitam.edu

(c) The Authors. Published by Blue Eyes Intelligence Engineering and Sciences Publication (BEIESP). This is an open access article under the CC BY-NC-ND license (http://creativecommons.org/licenses/by-nc-nd/4.0/)
The nodes communicate with each other within the given cluster or nodes within another cluster heads directly. Hence, in networking each node accomplish client and route functionalities simultaneously [11]. The design of the routing algorithms must integrate several factors owing to the MANETs features, e.g. node's trust, save energy, network topology dynamic changes. Considering the trust of node, the interaction of multi-hop nodes is relayed on the node's consistency of the route. Therefore, the existing node's trustworthiness is recognized significantly in the routing protocols. In MANETs, the capacity of the mobile node's battery is limited, which affects the survival of the network because the links are not connected while the battery is worn out. On considering the routing protocol, the energy of the mobile nodes is necessary for ensuring the network connectivity and enhances the life span of the network.

Factors that play a critical role in routing protocol

A.Optimal and Effective Routing: Which is one of the primary requirement of the defined protocol. We have to consider this as the primary goal of the designed protocol has to unearth and transfer the packets from the source to the destination. Packet Delivery Ratio is one of the parameter for efficient routing, end-to-end delay's average and optimal rout's percentage are also considered to make the routing protocol efficient and optimal.

B. Congestion Avoidance: It Strongly evaluated that congestion is linked with packet drops, if the congestion is more the packet drop rate will be more which decreases the efficiency of routing protocol.

C.Energy Consumption: Due to the increased smart devices and mobile nodes like PDA's, laptops, cellular phones and other portable devices are having strong demand towards energy consumption. To the analysis made by experts individual nodes energy consumption should be minimized.

D. Load Balancing: The nodes that located in the communication of optimal routes may suffer from overloads which might lead to network congestion. Fail distribution of load has to be attained between the individual nodes in optimal routs

E.Reachability: The probability of finding at least a single path between the selected source and destination nodes is considered as reachability

During routing, the flexibility of the nodes made it complex to identify the network topology utilized by nodes. Moreover, the volatile environment, the untrustworthy mobile nodes, and the wireless medium might cause different defects in MANETs.

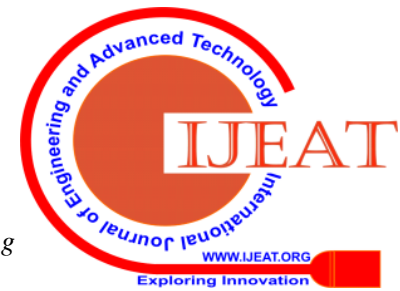




\section{Artificial Intelligence Based A* Optimization Routing In Mobile Ad Hoc Networks}

There are several suggestions on different strategies and protocols, as the "Internet Engineering Task Force and academic and industrial undertakings" are taking additional standardization efforts [24].

The conventional routing algorithm is categorized into three groups, such as proactive,

Hybrid, reactive routing on the basis of time triggered route discovery [13] [25]. Every node in the defined network will have global view and routing table is updated in certain time intervals through every node communicate its routing table with neighbours In proactive routing, every node communicates its route table occasionally for its neighbours to the extent that each node is having a global view of the network, for example PSR [14], and FSR [15]. Nodes create routes only on request in reactive routing such as AODV [23], and CAODV [16]. In general, hybrid routing separates the network into various regions, and then proactive routing is selected in the region and reactive routing between the regions, like ZRP [17], and HOPNET [13]. Excessive emphasis on routing trust leads to improve the control overhead and decrease the routing efficiency. At the same time overemphasis on routing efficiency refuses the additional features that might react to routing efficiency. Few existing methodologies are implemented to enhance the efficiency and routing trust i.e., cryptography and data hiding, location-aware and their combinations, trust value, and detection

The major contribution of the paper is to frame an optimized $\mathrm{A}^{*}$ algorithm. A* is one among the best optimization search algorithm in artificial intelligence. It makes use of heuristic values for path-finding. A* searching technique can be considered as one of the algorithm that follows smart technique which make it better than any conventional algorithms. The algorithm has been used to discover shortest path in some of the game designing and maps that are webbased very efficiently. The existence of obstructing obstacles in the network area challenges the design of routing protocols in both static and mobile ad hoc networks In A* Algorithm at each and every time it selects the node based on the value of ' $f$ ' which equal to the added value of $h$ and g. The node selection is done at every step by finding the node with lowest ' $f$ '. The ' $g$ ' and ' $h$ ' values are defined as

$\mathbf{g}=$ the cost incurred in forming a path from the source node to destination in the selected square of the grid. $\mathbf{h}=$ the cost incurred in forming a path from selected square grid, i.e., the current node to the destination node, we consider heuristic as a smart guess.

The organization of the paper is designed according to the details mentioned below:

- Section II reviews the features and challenges of conventional MANETs routing algorithms.

- $\quad$ Section III speaks about routing strategy in MANET: network and system model with proposed routing.

- Section IV in this section Obstacle aware routing pattern in MANET using A* algorithm is given.

- $\quad$ Section V illustrates simulation setup of the system

- Section VI Performance evaluation of routing protocols

- $\quad$ Section VII concludes this paper

\section{ROUTING STRATEGY IN MANET: NETWORK AND SYSTEM MODEL WITH PROPOSED ROUTING}

\section{A. System Model}

MANETs in general, consists of arbitrary and random movable platforms named nodes. The information pertains to the network is transmitted from one node to other through the major theme of the routing protocol in ad hoc networks is to create an effective and exact path between the nodes and delivers the messages in time. As there is restricted number of resources in MANETs, the protocols adjust themselves according to the alterations happen in the network status i.e., network size, and traffic density, and the resources are utilized efficiently. The network involves a flat network infrastructure. Moreover, a shared medium is available in the network, which is extremely demandable for the radio communication. The computer, or node or any device in the architecture could be referred to a router, or the end host, in which the nodes are generally autonomous.

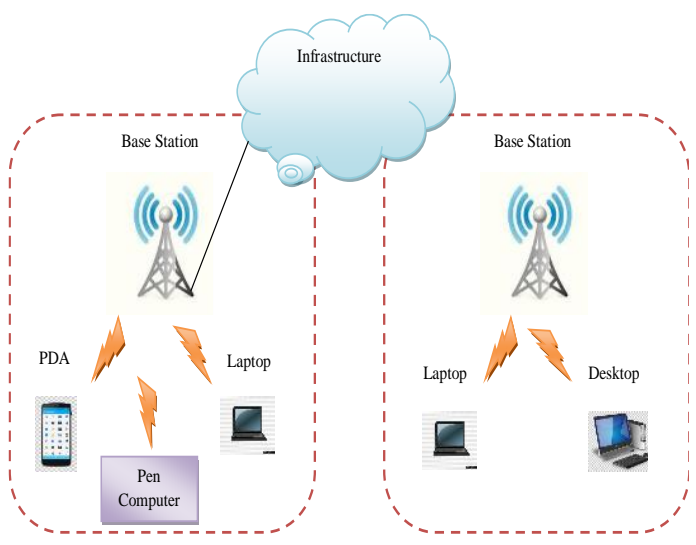

Fig. 1: General Architecture of MANETs

The development of wireless communication in MANET could be accomplished for the nodes that are adequately near to the sender i.e., within the sender's transmission range, the message is received with more signal strength. At the time of whole communication delay, the sender and the receiver will move out or in the network by not concerning the transmission range of sender.

\section{B. Communication Model}

MANET is a self-organizing structure, which consists of more wireless linked mobile devices. For the communication, each device can move in any direction, but should aware of the signal strength. Accordingly, the communication pattern of MANET is shown in Fig. 2. Here, the nodes that are present in the range of communication will communicate with each other, and the nodes that are far away from the communication range will follow the theory of multi-hop communication to communicate. 


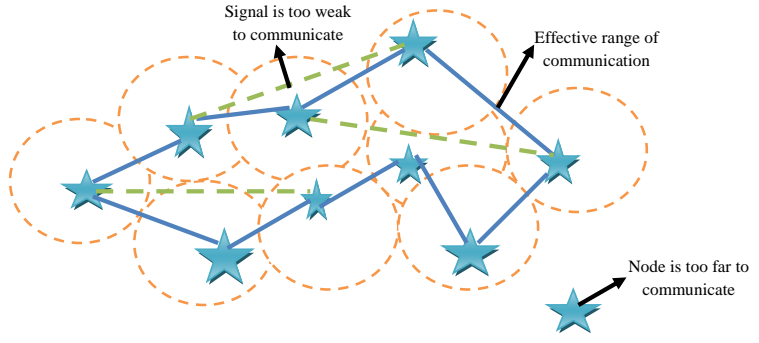

Fig. 2: Communication Pattern of MANET

One of the primary challenges in framing a MANET is enabling each device to incessantly sustain the data transmission without any traffic.

Since MANETs are a type of wireless ad hoc networks, it usually posses a routable networking environment on top of a Link Layer ad hoc network. Moreover, the routing Hence, it is highly necessary to find a novel structure the uniformly distributed in the network with area $M D \times N D$.

\section{Proposed Flow Graph for Obstacle Aware Routing in MANET}

In the past years, many researchers had dedicated their precious time for improving the efficiency of MANETs routing algorithms. The main intend of this paper is to develop obstacle-aware routing protocol in MANET using optimized $\mathrm{A}^{*}$ algorithm. The flow diagram of the proposed MANET routing is shown in Fig. 3.

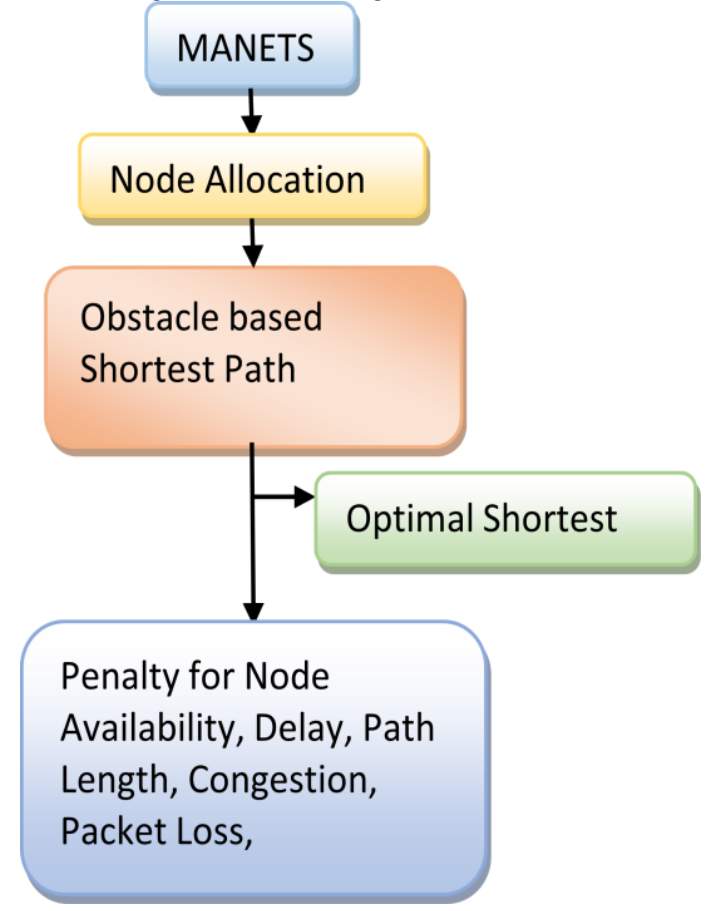

Fig. 3: Diagrammatic representation of proposed
MANET Routing

A*algorithm, which is a well-performing path selection algorithm by concerning the obstacles. Even though this algorithm is optimally efficient to solve complex routing problems, its performance has to be still improved by taking into account few more constraints other than node strategy often suffers from some obstacles, which need to be focussed for the development of improved routing desires. wireless network. Assume $N_{M}$ number of mobile nodes is

availability and distance. Hence, in order to further improve the performance of shortest route path selection by $A^{*}$ algorithm, an improved meta-heuristic algorithm termed as CE-CSO is deployed for optimally placing the obstacles in different locations for finding the optimal shortest path. Here, the improved shortest path selection is done in such a way that the total cost concerning the sum of the distance between the path and the congestion of each node in the network is minimized.

\section{OBSTACLE AWARE ROUTING PATTERN IN MANET USING A* ALGORITHM}

The obstacle aware shortest path is determined by the $A^{*}$ algorithm. In general, $A^{*}$ algorithm [29] is used to find the shortest paths from the source to destination by removing obstacles. Moreover, it is designed with a classical gridbased approach. For defining the process of $A^{*}$ to find the shortest path, the diagrammatic representation is given in Fig. 4

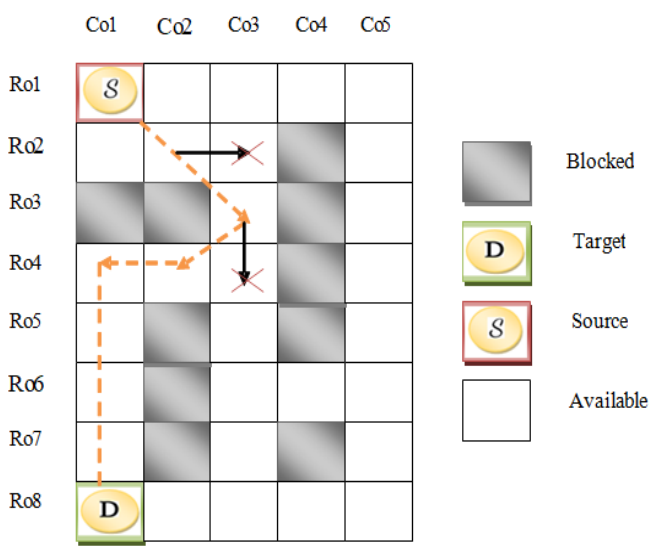

Fig. 4: Shortest path representation

In Fig. 4, the D the target node, obstacles are indicated by the black node, and the source node is denoted by the $\mathrm{S}$, and the other blocks are for the normal nodes. In the above diagram, $A^{*}$ algorithm chooses each step in an efficient manner i.e., the algorithm travels from (Ro1, Co1) to (Ro3, Co3) not to (Ro2, Co3). In addition, the algorithm moves from (Ro3, Co3) to (Ro4, Co2) but not to (Ro4, Co3). In the mean time, the squares have distance attribute separately consisting of $F(b), G(b)$, and $H(b)$ values. The cost $F(b)$ is the sum of $G(b)$ and $H(b)$, which is mathematically represented in Eq. (1). The value of $G(b)$ is the movement cost from source node to the destination node $(b)$ and the value of $H(b)$ is the movement cost from the current node (b) to the destination node(b).

$$
F(b)=G(b)+H(b)
$$

In order to reach the destination node from the source node in a shortest way, $\mathrm{A}^{*}$ algorithm is utilized. By using $A^{*}$ algorithm, the minimum $F$ value need to be found out. This procedure needs to be repeated until the shortest path is found with minimum cost. 


\section{Artificial Intelligence Based A* Optimization Routing In Mobile Ad Hoc Networks}

It is happened by maintaining the paths starting at the source node and moving towards the path end at a time until the termination criterion is met.

In Eq. (1), $b$ is the next node in the path. When A*selects the path to extend from source node to the target node, the $A^{*}$ search is terminated. For performing repeated selection of minimum cost nodes to extend, the applications of $\mathrm{A}^{*}$ makes use of priority queue. Moreover, the priority queue is called open set or fringe.

Here, at each step, the node consisting of low $F(b)$ value will be eradicated from the queue. The values of $F(b)$ and $G(b)$ of its neighbors are updated accordingly and those are added to the queue. The $\mathrm{A}^{*}$ continues its search until the destination node has minimum $F(b)$ value than any other node in the queue, and the corresponding value will be the cost of shortest path.

In an admissible heuristic, the value of $H(b)$ is zero at the the algorithm is easily revised, thus every node in the path has the information of predecessor node. This algorithm will run up to the destination node by pointing to the predecessor and so on, until few node's predecessor is the source node.

\section{A* Algorithm :}

Two list are defined as CLOSED list and OPEN list

1. Open list is initialized

2. Closed list is initialized

In the open list take the starting node(by marking ' $\mathrm{f}$ ' as zero)

3. Loop begins by checking for open list is empty or not

4. Node's least ' $\mathrm{f}$ ' value from open is selected, let it be 'q'

5. Pop ' $q$ '

6. 'n' q predecessor are generated

7. For every predecessor

i) if the goal is predecessor, search is stopped predecessor.g $=\mathrm{q} \cdot \mathrm{g}+$ (distance from q to predecessor $)$ predecessor.h $=$ distance from predecessor to goal predecessor.f $=$ predecessor.g + predecessor.h

ii) the predecessor is skipped, if the value of ' $\mathrm{f}$ ' is less than the predecessor at same position from OPEN list

iii) the predecessor is skipped, if the node of CLOSED list at same position as of predecessor has a lower $f$ value than predecessor, else to OPEN list add that node

$$
\text { end (for) }
$$

8. Into closed list $\mathrm{q}$ is pushed end (loop)

\section{Euclidean Distance :} destination node is considered as distance formula

- $\mathrm{H}=\operatorname{sqrt}\left((\text { current_node.x }-\operatorname{destination.x})^{2}{ }^{2}\right.$ (current_node - destination.y) $)^{2}$ )

- To find the path the signals are allowed to walk in any route, we can make use of heuristic values destination node. In order to define the exact series of steps,

- Heuristic value is the distance form the current node to

The determination of obstacle ware shortest path is done by the $A^{*}$ algorithm, in which the node availability and distance between the path is considered. Here, the obstacle is recognized by the CE-OLSR [35]. The dynamic and automatic detection of obstacles that present in the network is accomplished by CE-OLSR with no extra signalling overhead. It is an advanced version of OLSR protocol, which depends on two concepts like "network cartography, and stability routing". In order to build an accurate network topology, CE-OLSR utilizes network cartography as a substitute to link states. For avoiding the usage of weak links during the routing procedure, CE-OLSR depends on stability routing.

\section{SIMULATION SETUP OF THE SYSTEM}

The simulation setup involves network basic settings the simulation is performed in Matlab simulator.

The below table gives the considered parameter values for the proposed A* algorithm

Table I : Routing Parameters

\begin{tabular}{|ll|}
\hline \multicolumn{1}{|c}{ Parameters } & \multicolumn{1}{c|}{ Value } \\
\hline Number of nodes & Varies \\
\hline Mac Type & $802.11 \mathrm{e}$ \\
\hline Protocol & AODV, A* \\
\hline Communication Protocol & UDP \\
\hline Application & CBR \\
\hline Delay & $1 \mathrm{~ms}$ \\
\hline Simulation Time & Varies \\
\hline
\end{tabular}

\section{PERFORMANCE EVALUATION OF ROUTING PROTOCOLS}

This design focuses on the specified metrics performance and are measured quantitatively. The accuracy analyzed over the designed algorithm, performance metrics plays a main role. The metrics that are considered are as follows,

A. Delay: The average time spend for the transmission of a packet from source to destination point. The Total_Delay_of_Packets at Destination_Node is $d_{i}$, and the Total_Number_of_Packets_Received at the destination is $p k t d_{i}$. n's Traffic iThe results of the proposed algorithm

B.Path Length: Path length is considered as the distance that is measured from the source node to the destination node.

C. Congestion Cost: Congestion cost is considered as the cost that is charged due to the problem of congestion

D. Penalty for Node Availability: The main criteria of laying the path is the availability of nodes. if more time is taken in finding the availability of the node, the over-all outcome may be effected. this delay in identifying the neighbouring nodes is termed as penalty of node availability. Path finding time is effected by the node penalty. 
E. Packet Loss: when the data packet are transmitted through the network path in selected route when any packet doesn't reach the destination point i.e the packet is dropped in the middle before the reach of destination then the loss of packet occurs. Packet loss is also proportional to the congestion in the network. Loss of Packet loss is calculated as the percentage of lost packets of sent packets.

F.Throughput: The count of bits transmitted per unit of time from source point to the destination point.

\section{SIMULATION RESULTS}

\section{About MATLAB simulator}

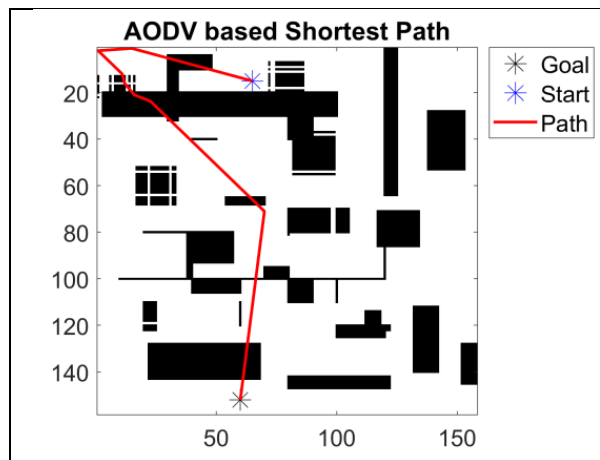

For technical computations and attain high-performance the language MATLAB can be used. We can find integration of visualization, as an easy computation environment, visualization and mathematical notations can be might lead to the solutions that are familiar. The other uses can discussed as the, Development of algorithm, Computation and Math, Simulation, prototyping, modelling the system, And many more

The below result are obtained by implementation of the A* algorithm in the MATLAB

While considering the network with obstacle the how the signal propagation is being taken place and based on which the route formed is depicted in below figures 5 and 6 for AODV and A* algorithms respectively.

\section{Fig. 5 The path finding in obstacle aware network with AODV and A*}

The below tables depict the performance of two routing protocol i.e., the conventional routing protocol AODV and proposed obstacle aware optimal artificial intelligence routing protocol $\mathrm{A}^{*}$, where the network of light and dense are being considered.

The following simulation results elucidates the comparison between conventional AODV and $\mathrm{A}^{*}$ algorithm. When AODV algorithm is applied for packet routing the

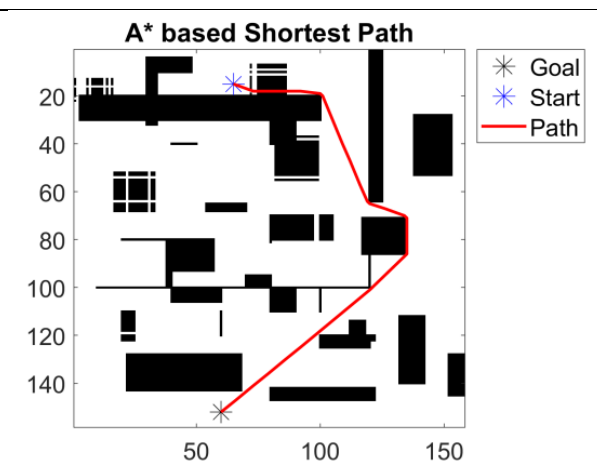

TABLE II. PERFORMANCE ANALYSIS OF PROPOSED AND CONVENTIONAL OBSTACLE-AWARE MANET ROUTING FOR 78, 108, 128, 200 NODES

\begin{tabular}{|c|c|c|c|c|c|}
\hline Measures For 78 Nodes & AODV & $\mathbf{A}^{*}$ & Measures For 108 Nodes & AODV & $\mathbf{A}^{*}$ \\
\hline Path Length & 176.29 & 67.52 & Path Length & 194.29 & 130.03 \\
\hline Congestion Cost & 15.882 & 25.638 & Congestion Cost & 2.1272 & 40.416 \\
\hline Penalty for Node Availability & 160 & 0 & Penalty for Node Availability & 220 & 0 \\
\hline Delay & 0.71 & 0.35 & Delay & 0.71 & 0.68 \\
\hline Packet Loss & 0.1 & 0.34 & Packet Loss & 0.08 & 0.69 \\
\hline Throughput & 126.76 & 94.286 & Throurahnut & 129.58 & 22.794 \\
\hline Total Cost & 352.99 & 93.859 & & & \\
\hline & & & Total Cost & 417.22 & 171.86 \\
\hline
\end{tabular}


Artificial Intelligence Based A* Optimization Routing In Mobile Ad Hoc Networks

\begin{tabular}{|c|c|c|c|c|c|}
\hline Measures For 128 Nodes & AODV & $\mathbf{A}^{*}$ & Measures For 200 Nodes & AODV & $\mathbf{A}^{*}$ \\
\hline Path Length & 217.36 & 186.39 & Path Length & 247.45 & 217.22 \\
\hline Congestion Cost & 9.269 & 44.007 & Congestion Cost & 16.943 & 37.1 \\
\hline Penalty for Node Availability & 220 & 0 & Penalty for Node Availability & 170 & 0 \\
\hline Delay & 0.71 & 0.61 & Delay & $0_{5}$ & - 7 \\
\hline Packet Loss & 0.07 & 0.62 & & & \\
\hline & & & Packet Loss & 0.06 & 0.75 \\
\hline Throughput & 130.99 & 31.148 & & & \\
\hline Total Cost & 44741 & 23166 & Throughput & 144.62 & 16.892 \\
\hline & & & Total Cost & 435.11 & 255.87 \\
\hline
\end{tabular}

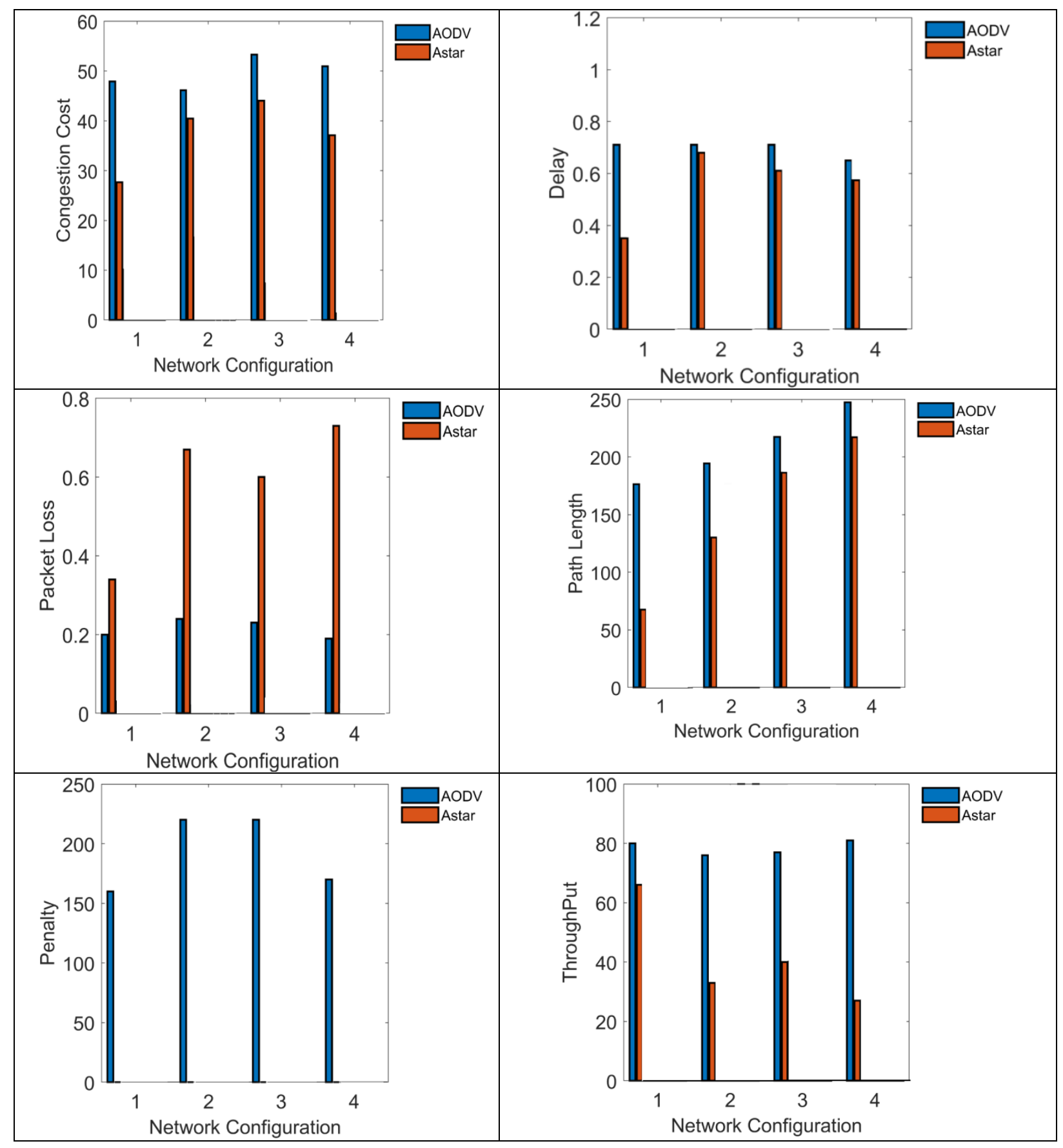




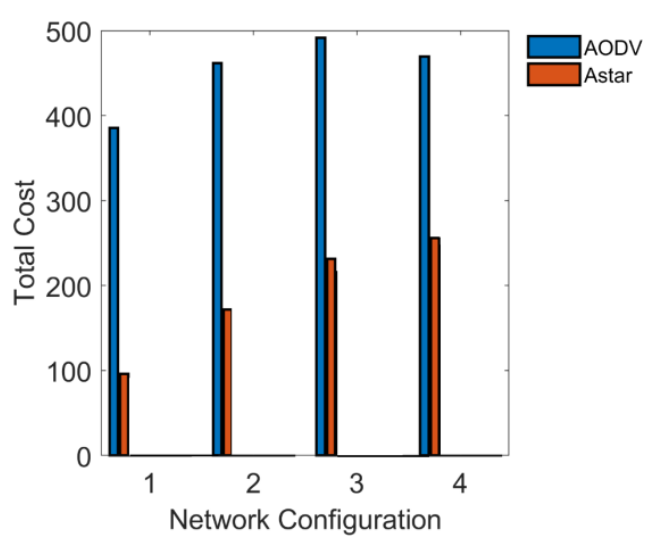

\section{CONCLUSION}

The A* signalling, can be used for automatic presumption of the occurrences of static obstacles positioned in the network. This work identifies the obstacles and lays the shortest path optimally. Simulation results reveals that defined metrics improves the accuracy and coverage ratio. The attained results shows that the proposed algorithm improves the effectiveness by optimally recognising the obstacle area and minimizing the path laying cost and penalty of node availability. The proposed work provides a sufficient accuracy ratio to economically full fill our aim to autonomously avoid broken links due to obstacle obstruction. The proposed obstacle detection algorithm is integrated with CE-OLSR

\section{REFERENCES}

1. Sanjeev Kumar Sharma, et al., "Improvement over AODV Considering QoS Support in Mobile Ad-hoc Networks" 21 March 2017. International Journal of Computer Networks and Applications (IJCNA).DOI: 10.22247/ijcna/2017/48967

2. R. M. Chintalapalli et al., multi-objective optimisation model for secure routing in mobilead-hocnetwork," IET Communications, vol. 12, no. 12, pp. 1406-1415, 3172018.

3. Subhrapratim Nath, et al., " Optimizing MANET routing in AODV : An Hybridization approach of ACO and Firefly Algorithm" 978-15090-1047-9/16/\$31.00 (C)2016 IEEE.

4. P. Francis Antony Selvi et al., "Ant based multipath backbone routing for load balancing in MANET," IET Communications, vol. 11, no. 1, pp. 136-141, 512017.

5. Xiang Ji, X. J. Li et al., " Efficient and Reliable Cluster-Based Data Transmission for Vehicular Ad Hoc Networks" Mobile Information Systems Volume 2018, Article ID 9826782.

6. M. Malathi, et al., "Modified Bi-directional Routing with Best Afford Path (MBRBAP) for Routing Optimization in MANET", Wireless Personal Communications, vol.90, no.2, pp 861-873, September 2016.

7. Hua Yang, et al., "A method of routing optimization using $\mathrm{CHNN}$ in MANET", Journal of Ambient Intelligence and Humanized Computing, vol.10, no.5, pp 1759-1768, May 2019.

8. I. Kacem, et al., "A New Routing Approach for Mobile Ad Hoc Systems Based on Fuzzy Petri Nets and Ant System," IEEE Access, vol. 6, pp. 65705-65720, 2018.

9. Wei Quan, et al., "Content retrieval model for information-center MANETs: 2-dimensional case," 2013 IEEE Wireless Communications and Networking Conference (WCNC), Shanghai, 2013, pp. 4422-4427.

10. Kohei Arai, et al., "Decision Making and Emergency Communication System in Rescue Simulation for People with Disabilities", International Journal of Advanced Research in Artificial Intelligence, vol.2, no.3, March 2013.

11. WolfgangKiess, et al., "A survey on real-world implementations of mobile ad-hoc networks", Ad Hoc Networks, vol.5, no.3, pp.324-339, April 2007.
12. Jieying Zhou, et al., "Ad Hoc On-Demand Multipath Distance Vector Routing Protocol Based on Node State", Communications and Network, no.05, vol.03, pp.408-413, January 2013.

13. JianpingWang, et al., "HOPNET: A hybrid ant colony optimization routing algorithm for mobile ad hoc network", Ad Hoc Networks, vol.7, no.4, pp.690-705, June 2009.

14. Z. Wang, et al., "PSR: A Lightweight Proactive Source Routing Protocol For Mobile Ad Hoc Networks," IEEE Transactions on Vehicular Technology, vol. 63, no. 2, pp. 859-868, Feb. 2014.

15. Guangyu Pei, et al., "Fisheye state routing: a routing scheme for ad hoc wireless networks," 2000 IEEE International Conference on Communications. ICC 2000. Global Convergence Through Communications. Conference Record, New Orleans, LA, USA, vol.1, pp. 70-74, 2000.

16. Angela SaraCacciapuoti, et al., "Reactive routing for mobile cognitive radio ad hoc networks", Ad Hoc Networks, vol.10, no.5, pp.803-815, July 2012.

17. M. R. Pearlman et al., "Determining the optimal configuration for the zone routing protocol," IEEE Journal on Selected Areas in Communications, vol. 17, no. 8, pp. 1395-1414, Aug. 1999.

18. G. Zhan, W. Shi et al., "Design and Implementation of TARF: A Trust-Aware Routing Framework for WSNs," IEEE Transactions on Dependable and Secure Computing, vol. 9, no. 2, pp. 184-197, March-April 2012.

19. F. Bao, I. Chen, et al., "Hierarchical Trust Management for Wireless Sensor Networks and its Applications to Trust-Based Routing and Intrusion Detection," IEEE Transactions on Network and Service Management, vol. 9, no. 2, pp. 169-183, June 2012.

20. HuiXia, et al., "Trust prediction and trust-based source routing in mobile ad hoc networks", Ad Hoc Networks, vol.11, no.7, pp.20962114, September 2013

21. F. Kuhn, et al., "An Algorithmic Approach to Geographic Routing in Ad Hoc and Sensor Networks," IEEE/ACM Transactions on Networking, vol. 16, no. 1, pp. 51-62, Feb. 2008.

22. L. Zhou, et al., "Energy-Spectrum Efficiency Tradeoff for Video Streaming over Mobile Ad Hoc Networks," IEEE Journal on Selected Areas in Communications, vol. 31, no. 5, pp. 981-991, May 2013.

23. J. J. Ferronato et al., "Analysis of Routing Protocols OLSR, AODV and ZRP in Real Urban Vehicular Scenario with Density Variation," IEEE Latin America Transactions, vol. 15, no. 9, pp. 1727-1734, 2017.

24. Z. Zhu, et al., "Dynamic Service Provisioning in Elastic Optical Networks With Hybrid Single-/Multi-Path Routing," in Journal of Lightwave Technology, vol. 31, no. 1, pp. 15-22, Jan.1, 2013.

25. X. Li, Z. Jia, et al., "Trust-based on-demand multipath routing in mobile ad hoc networks," IET Information Security, vol. 4, no. 4, pp. 212-232, December 2010.

26. Kaur D, et al., Comparative analysis of AODV, OLSR, TORA, DSR and DSDV. International Journal Computer Network and Information Security. 2013; 5(3):39-46

27. Siakoulis Y, et al., The impact of simulation duration on the performance of the OLSR, AODV and DSDV Protocols, in a heavyloaded Ad-hoc wireless mobile environment. First IEEE International Conference on System Informatics and Modeling, Greece. 2014. p. 160-7.

28. Barinder Singh, et al., "To Propose Enhancement in Reactive Routing AODV Protocol to Overcome Congestion in MANET", Vol. 4, Issue. 9, pg.296 - 303,September 2015.

29. LeiXie, et al, "A path planning approach based on multi-direction A* algorithm for ships navigating within wind farm waters", Ocean Engineering, vol.184, pp.311-322, 15 July 2019.

30. M.E.H.Pedersen, et al., "Simplifying Particle Swarm Optimization", Applied Soft Computing, vol.10, no.2, pp.618-628, March 2010.

31. A.H.Gandomi, et al., "Firefly algorithm with chaos", Communications in Nonlinear Science and Numerical Simulation, vol.18, no.1, pp.89-98, January 2013.

32. SeyedaliMirjalili et al., "Grey Wolf Optimizer", Advances in Engineering Software, vol.69, pp.46-61, March 2014.

33. SeyedaliMirjalili, et al., "The Whale Optimization Algorithm", Advances in Engineering Software, vol.95, pp.51-67, May 2016. 
34. Luminita Moraru, et al., "Geographic Routing with Early Obstacles Detection and Avoidance in Dense Wireless Sensor Networks" ADHOC-NOW 2008, LNCS 5198, pp. 148-161, 2008. SpringerVerlag Berlin Heidelberg 2008

35. Abdelfettah Belghith, et al., "Autonomic Obstacle Detection and Avoidance in MANETs Driven by Cartography Enhanced OLSR", Mobile Information Systems, Volume 2015.

\section{AUTHORS PROFILE}

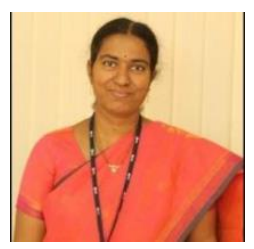

Mrs. G. Yasoda Devi, (Ph.D), research scholar, department of Computer Science and Engineering, GIT, GITAM Deemed to be university, Visakhapatnam. Working as assistant professor in the Department of Computer Science and Engineering, Lendi Institute of Engineering and Technology, Jonnada, Vijayanagaram. My interested areas of research are Networks,

MANETs, Machine Learning.

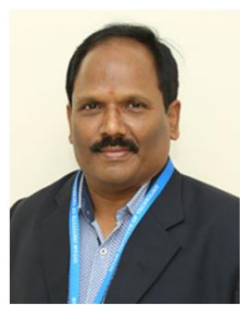

Dr. Gurrala Venkateswara Rao, Professor, Department of CSE, GIT, GITAM Deemed to be university, Visakhapatnam, Andhra Pradesh. Research areas of interest include Networks, Mobile Computing and Software Engineering. Around 50 papers have been published in various conferences and journals. Five Ph.D's have been awarded under his guidance. He also completed a major UGC project. 